\title{
BLICKDIAGNOSE-QUIZ
}

\section{Was ist das für ein Fleck am Bauch?}

Auflösung von Seite 5

Bei dem Befund handelt es ich um eine zirkumskripte Sklerodermie, wie die histologische Untersuchung einer Probeentnahme ergab. Die Sklerodermie gehört zu den Kollagenosen und somit zu den rheumatisch-entzündlichen Erkrankungen.

\section{Es werden zwei Untergruppen unterschieden:}

(1) Die systemische Sklerodermie. Diese bezieht auch das Gefäßsystem und innere Organe mit ein. Hierdurch können erhebliche Funktionsstörungen und Beschwerden ausgelöst werden. Insbesondere die Lunge, die Nieren und der Verdauungstrakt sind betroffen.

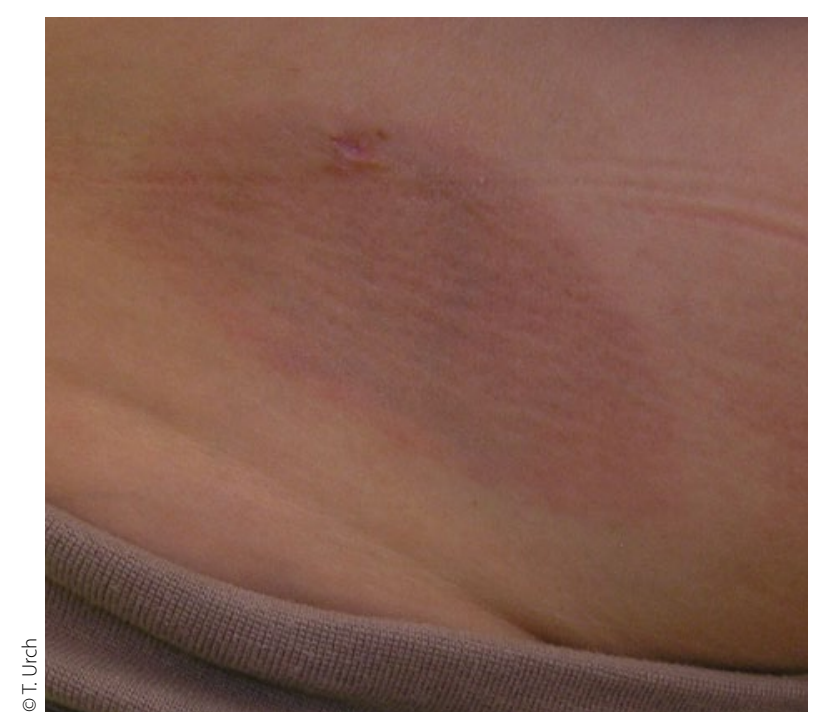

Zirkumskripte Sklerodermie.
2 Die zirkumskripte Sklerodermie. Diese ist auf das Bindegewebe der Haut beschränkt. Hierbei werden verschiedene Untergruppen unterschieden. Am häufigsten tritt die limitierte Form auf (auch Plaqueform oder Morphea genannt). Diese findet sich an druckexponierten Stellen, insbesondere am Übergang der Hüft- in die Inguinalregion. Initial tritt ein meist ovales Erythem auf, das sich langsam vergrößert. Später tritt im Zentrum eine elfenbeinfarbene Verhärtung (Plaque) auf. Im Verlauf kommt es häufig zu einer postinflammatorischen Hyperpigmentierung der betroffenen Areale.

Es gibt keinen Übergang von der zirkumskripten zur systemischen Sklerodermie.

Eine eindeutige einzelne Ursache für das Erkrankungsbild konnte bisher nicht nachgewiesen werden. Verschiedene Therapieformen können das Voranschreiten der Erkrankung, insbesondere bei der systemischen Sklerodermie, zumindest verlangsamen. Bei der zirkumskripten Form ist allerdings eine spezielle Therapie meist nicht erforderlich.

\section{Keyword: scleroderma}

\section{- Dr. med. Thomas Urch \\ Allgemeinmedizin \\ Am Rosengarten 6 \\ D-25554 Wilster}

